\title{
LA METAPOBLACIÓN DEL VENADO BURA EN LA RESERVA DE LA BIOSFERA MAPIMÍ, MÉXICO: CONSIDERACIONES PARA SU CONSERVACIÓN
}

\section{G. Sánchez $\operatorname{Rojas}^{1}$ y S. Gallina ${ }^{2}$}

1 Centro de Investigaciones Biológicas, Universidad Autónoma del Estado de Hidalgo gsanchez@uaeh.edu.mx

2 Departamento de Ecología y Comportamiento Animal, instituto de Ecología, A. C. sonia.gallina@inecol.edu.mx

\section{LA HETEROGENEIDAD ESPACIAL DE LAS POBLACIONES}

En la actualidad entendemos que el entorno ecológico tiene patrones espaciales y es discontinuo, variando a lo largo del tiempo de una forma compleja y muchas veces impredecible, por lo que es heterogéneo y en estado de no equilibrio (SPARROW, 1999). LEVIN (1992) captura esta visión al declarar que "el problema de los patrones y las escalas son el problema central en la ecología" por lo que todos los sistemas ecológicos exhiben heterogeneidad y fragmentación en una amplia gama de escalas y esta fragmentación es fundamental para la dinámica de las poblaciones, la organización de las comunidades y el ciclo de los elementos en un ecosistema.

En el caso de la dinámica de las poblaciones, se reconoce que el hábitat de muchas especies es hete- rogéneo a diversas escalas debido tanto a procesos naturales como a las actividades humanas (LORD \& NORTON, 1990). Por lo que indudablemente las poblaciones están estructuradas espacialmente como el ambiente en el que viven, el cuál usualmente es heterogéneo, por lo que estas poblaciones tienden a responder a esta heterogeneidad de alguna manera (SZACKI, 1999).

ANDREWARTHA \& BIRCH (1984) simplifican esta estructura espacial en dos escalas relevantes para comprender la dinámica de la población de cualquier especie, la escala local (resolución fina) y la escala natural de las poblaciones (resolución gruesa o regional). La escala local es aquella en que los miembros de esa población tienen más probabilidades de aparearse entre sí que individuos de otras poblaciones (ANDREWARTHA \& BIRCH, 1984). Debido a los disturbios y a la variabilidad demográfica los conjuntos de individuos 


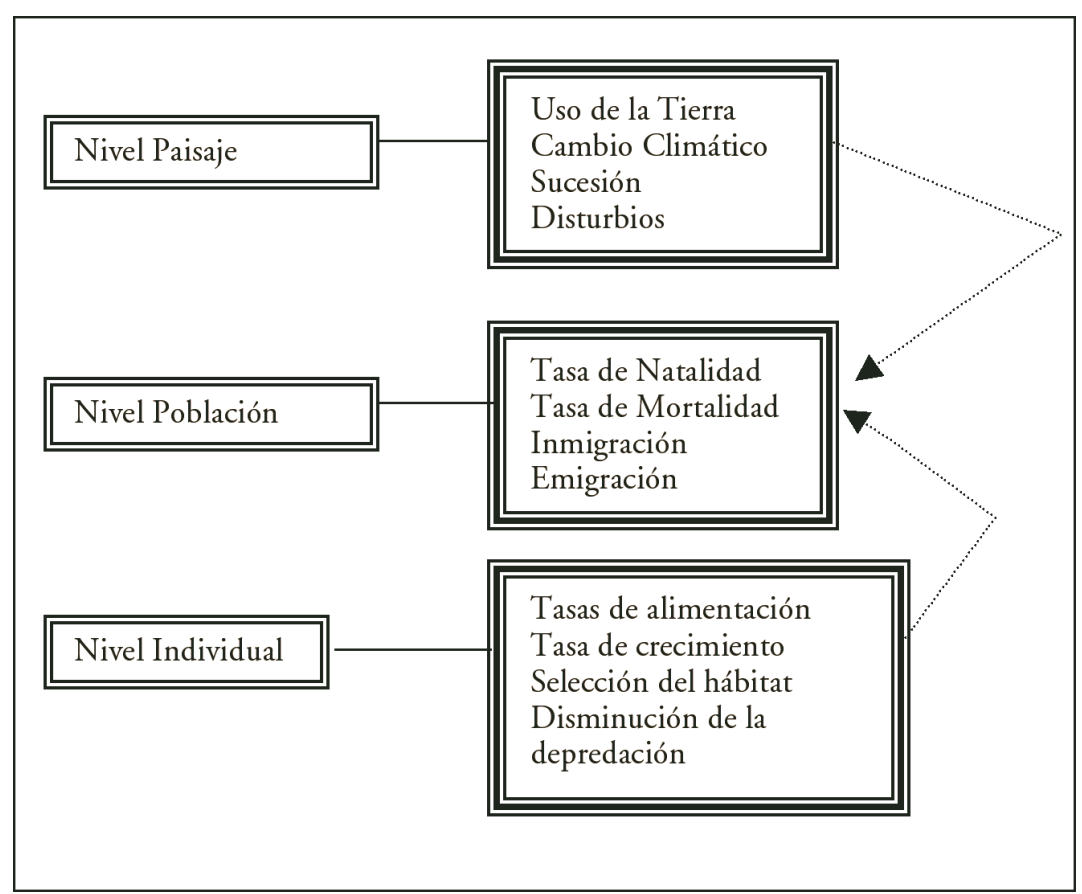

Figura 1. La dinámica de las poblaciones deberá ser comprendida como el resultado de un proceso jerárquico que afecta a la población a diferentes niveles. El nivel del paisaje cambia la disponibilidad de fragmentos adecuados para una especie determinada. La disponibilidad de fragmentos adecuados y el comportamiento y fisiología de los individuos se combina para influir en la dinámica de las poblaciones.

de una población local (que llamaremos desde ahora subpoblación) a menudo tiende a la extinción o desaparición temporal. Por lo que para que las poblaciones tengan permanencia en escalas regionales se necesita que en las áreas donde se haya extinto la especie sean colonizadas por individuos de otras áreas mediante la dispersión (FAHRIG \& MERRIAM, 1994). Esto nos obliga a percibir a la dinámica de las poblaciones como un proceso jerárquico, como se muestra en la Figura 1 (PULLIAM \& DUNNING, 1994).

A partir del reconocimiento de poblaciones subdivididas se han propuesto diferentes modelos teóricos para explicar su dinámica. El modelo más utilizado es el de metapoblación, el cual está en evolución constante por lo que se encuentran diversas definiciones de lo que es una metapoblación en la literatura (ALVAREZ-BUYLLA et al., 1996; GILPIN \& HANSKI, 1991; HANSKI \& GILPIN, 1991; HANSKI \& GAGGIOTTI, 2004) que tratan de explicar este patrón discontinuo variando en aspectos derivados de las capacidades de dispersión de los organismos utilizados como de los mecanismos que explican su dinámica interna. Dentro de este concepto en evolución para los grandes mamíferos quizás la definición de metapoblación propuesta por MCCULLOUGH (1996a) es la mejor aproximación dado que pone énfasis en aspectos biológicos que comparten mucho de ellos, de ahí que "una metapoblación es una población local que presenta una distribución discontinua. Esta se distribuye sobre fragmentos disyuntivos de hábitat adecuado separados por parches de hábitats inadecuados dentro de los cuales los animales no pueden establecerse. Debido al riesgo de mortalidad que representa el cruzar las condiciones hostiles de la matriz, el movimiento de los animales entre los parches no es rutinario; consecuentemente el movimiento entre fragmentos (dispersión) es restringido". En este escenario es muy probable que muchos fragmentos adecuados sean pequeños y soporten pequeñas poblaciones, por lo que la extinción en estos parches (extinción local) puede ser un evento común. Por lo tanto, la persistencia de la metapoblación depende de la dinámica de extinción y recolonización entre los diferentes fragmentos a través de la dispersión. Si la tasa de recolonización excede a la tasa de extinción, la metapoblación puede persistir aunque ninguna subpoblación puede sobrevivir en un fragmento particular a lo largo del tiempo (MCCULLOUGH, 1996b). 


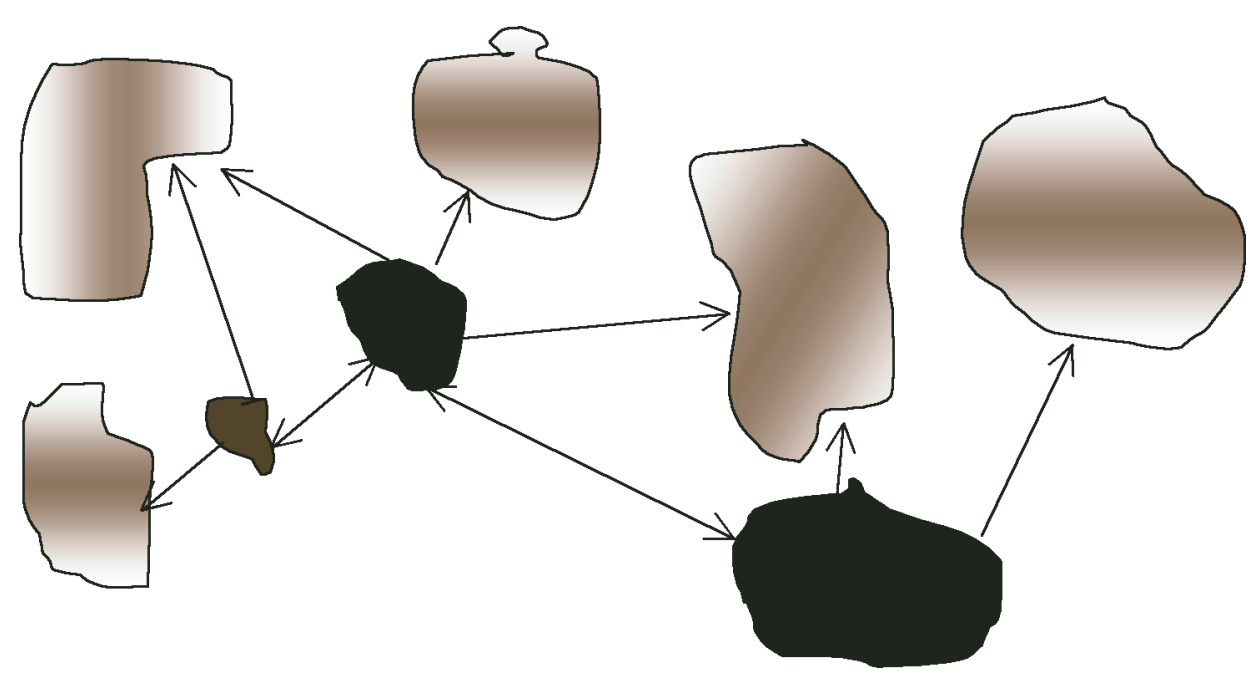

Figura 2. Ejemplo esquemático de la estructura de una metapoblación afectada por fragmentos fuente y fragmentos vertedero. En este caso unos cuantos fragmentos fuente (negro) proveen de un excedente de individuos los cuales emigran y colonizan los fragmentos vertedero (degradado). Los fragmentos vertedero pueden ser espacialmente mayores que los fragmentos fuente, y pueden incluso tener una densidad poblacional mayor, pero estas poblaciones rápidamente se extinguirían sin la presencia de los fragmentos fuente. En este esquema el paisaje estaria integrado por fragmentos negros, degradados y el área blanca representaria la matriz hostil. Las flechas indican la dirección mas probable de los movimientos de los individuos.

Los modelos teóricos también han servido para enfatizar el hecho de que en algunas poblaciones los individuos ocupan fragmentos de diferentes calidades, en donde se asume que los individuos que viven en un fragmento de mayor calidad producen un mayor número de descendientes, mientras que los individuos que utilizan fragmentos poco productivos son menos exitosos. Al modelo que explica la dinámica de la población en función de la calidad relativa de los fragmentos es conocido como la dinámica fuente-vertedero, una representación gráfica de esta dinámica se observa en la Figura 2 (PULLIAM \& DUNNING, 1994). A estos fragmentos productivos se les puede llamar fuentes y están definidos como áreas donde el éxito reproductivo es mayor que la mortalidad localmente. Mientras que en los fragmentos menos productivos el éxito reproductivo es menor que la mortalidad, a estos fragmentos los llamaremos vertederos, porque sin la inmigración de otras áreas, la población de estos fragmentos inevitablemente harán una espiral para caer en el vertedero de la extinción (PULLIAM, 1988; PULLIAM \& DANIELSON, 1991; PULLIAM \& DUNNING, 1994).
La heterogeneidad espacial entonces se identifica como un factor causal que afecta los procesos biológicos (LEVIN, 1992). Para comprender cómo actúa esta heterogeneidad es necesario estudiar los fenómenos biológicos usando una escala espacial de paisaje que comprenda toda esta heterogeneidad. El paisaje se define como un área de tierra heterogénea compuesta de un conjunto de ecosistemas interactuantes que se repiten a lo largo del área de forma semejante (FORMAN \& GODRON, 1986; FARINA, 1998). El arreglo espacial de los fragmentos, sus diferentes cualidades, su yuxtaposición y la proporción de los diferentes tipos de ecosistemas son elementos que modifican la dinámica de poblaciones y comunidades (FARINA, 1998).

A las poblaciones entonces hay que estudiarlas reconociendo la heterogeneidad que se presenta a diferentes escalas dentro del área donde se distribuyen y con un conocimiento profundo de la historia natural de la especie. Esto es particularmente importante si además podemos hacerlo dentro de un área protegida como las reservas, ya que muchas de ellas fueron creadas y concebidas mucho antes de que se reconociera la importancia de la heterogeneidad. 


\section{RESERVA DE LA BIOSFERA MAPIMÍ}

En los años setenta los especialistas en conservación agrupados en el Programa del Hombre y la Biosfera (MAB) de la UNESCO, preocupados por la ineficacia de los sistemas de protección del medio natural en los países en desarrollo, generaron un nuevo concepto de protección que combinaba cuatro elementos fundamentales: (1) la investigación científica, (2) la necesidad de tener áreas bien protegidas (llamadas zonas núcleo), (3) la participación local y (4) la aceptación de que los decretos oficiales de zonas amplias tenían ciertas ventajas que debían aprovecharse en un nuevo tipo de área natural protegida: la Reserva de la Biosfera (HALFFTER, 1985). Este concepto fue elaborado en 1974 y ya en 1976 existía una red de reservas de la biosfera en todo el mundo, y su crecimiento ha sido espectacular (HALFTTER et al., 2001).

Actualmente se reconoce como reservas de la biosfera a "zonas de ecosistemas terrestres o costeros/marinos, o una combinación de los mismos, reconocida en el plano internacional como tales en el marco del programa MAB-UNESCO”. Estas reservas son propuestas por los gobiernos nacionales y deben cumplir un mínimo de condiciones para ser admitidas. Las reservas de la biosfera tienen tres funciones fundamentales: una función de conservación para proteger los recursos genéticos, las especies, los ecosistemas y los paisajes; una función de desarrollo económico y humano sostenible; y una función de apoyo logístico para respaldar y alentar actividades de investigación, de educación, de formación y de observación permanente relacionadas con las actividades de interés local, nacional y mundial encaminadas a la conservación y el desarrollo sostenible (UNESCO 1996).

Cada reserva deberá contener tres elementos: 1) una o más zonas núcleo que beneficien la protección a largo plazo y permitan conservar la diversidad biológica; 2) una zona de amortiguación bien definida que generalmente circunde las zonas núcleo, que se utilice para actividades compatibles con prácticas ecológicas racionales; 3) una zona de transición flexible donde se realicen varias actividades productivas y en donde las organizaciones con interés en el medio ambiente trabajen conjuntamente en la administración y el desarrollo sostenible de los recursos de la zona (UNESCO 1996).
Este planteamiento general fue desde un principio ajustado a la realidad socioeconómica y política particular de cada país, por lo que conservando los postulados y objetivos base, no existe un esquema único de reserva de la biosfera (HALFFTER, 1984). En el caso de México dos reservas de la biosfera fueron pioneras en este programa, Mapimí y la Michilía, ambas en el Estado de Durango. En estas reservas se pone en práctica lo que se ha llamado la "Modalidad Mexicana" de las reservas de la biosfera e incluye cuatro lineamientos: 1) incorporación de la población e instituciones locales a la tarea común de la conservación del germoplasma; 2) incorporación de la problemática socioeconómica regional a los trabajos de investigación; 3) dar a las reservas una independencia administrativa, encargando de su gestión a instituciones de investigación que respondan ante las más altas autoridades del país; 4) considerar que las reservas deben formar parte de una estrategia global (HALFFTER, 1984).

La Reserva de la Biosfera Mapimí se ubica entre los $104^{\circ} 03^{\prime}$ y $103^{\circ} 32^{\prime}$ latitud $E$ y los $26^{\circ} 48^{\prime}$ y $26^{\circ} 31^{\prime}$ longitud N. El área presenta un clima seco y extremoso semi-cálido con lluvias de verano. La precipitación suele ser en forma de violentos chubascos de corta duración. El promedio anual de precipitación es de $264.2 \mathrm{~mm}$ con una máxima de $513 \mathrm{~mm}$ y una mínima de $81 \mathrm{~mm}$. La temperatura media anual es de $20.8^{\circ} \mathrm{C}$ con una mínima promedio en el invierno de $3.9^{\circ} \mathrm{C}$ y una máxima en el verano de $36.1{ }^{\circ} \mathrm{C}$ (Cornet 1988). La reserva originalmente tenía una extensión de $1720 \mathrm{~km}^{2}$ presentando 7 elementos del paisaje: bajadas y lomas de origen ígneo y sedimentario (37\% del área); bajadas y sierras de origen calcáreo (17\%); playa sur (18\%); playa norte $(6 \%)$; dunas (13\%); transición eólico-fluvial (8\%); mesa de basalto (1\%) (Montaña \& Breimer 1988). Actualmente, según el nuevo decreto (22 de noviembre del 2000), la reserva tiene $3424 \mathrm{~km}^{2}$.

\section{EL VENADO BURA}

El venado bura (Odocoileus hemionus) es una de las dos especies del genero Odocoileus que se reconocen en la actualidad, la otra es el venado cola blanca $(O$. virginianus) (Figuras 3 y 4 respectivamente). La especie se caracteriza por presentar un tamaño de cuerpo entre 1300 y $2600 \mathrm{~mm}$ de longitud total, una cola entre 115 
y $190 \mathrm{~mm}$ un peso de los machos de entre 64 y 114 $\mathrm{kg}$ y de las hembras entre 45 y $75 \mathrm{~kg}$ (LEOPOLD, 1965). En los machos se presentan astas con protuberancia sub-basal corta, el tronco principal se corta hacia arriba y afuera, y las puntas son dicotómicas del mismo tamaño; la cola tiene la punta negra y con tonos que van de blanco a negro en la parte dorsal; las orejas presentan en ambos sexos bordes obscuros y miden $2 / 3$ de la longitud de la cabeza; y presenta un escudo anal de color blanco en los cuartos traseros (Figura 5) (ANDERSON \& WALLMO, 1984).

Anteriormente se asignaba a la subespecies $O . h$. crooki las poblaciones de venado bura que habitan los desiertos de Sonora y en el interior del norte de México con sus regiones desérticas y semi-desérticas de vegetación abierta (LEOPOLD, 1965; WALLMO, 1981). Sin embargo, trabajos recientes reconocen que la descripción original de esta subespecie crooki se basó en un ejemplar híbrido entre cola blanca y bura por lo que es un sinónimo de O.h. eremicus (HEFFELFINGER, 2000). A pesar de esta controversia taxonómica se puede considerar que la distribución histórica de las poblaciones de venado bura en el desierto Chihuahuense de México, incluía totalmente el estado de Coahuila, el extremo sudoeste del estado de Nuevo León, la región norte de San Luis Potosí, una pequeña región en Tamaulipas, la región desértica de los estados de Chihuahua y Du-

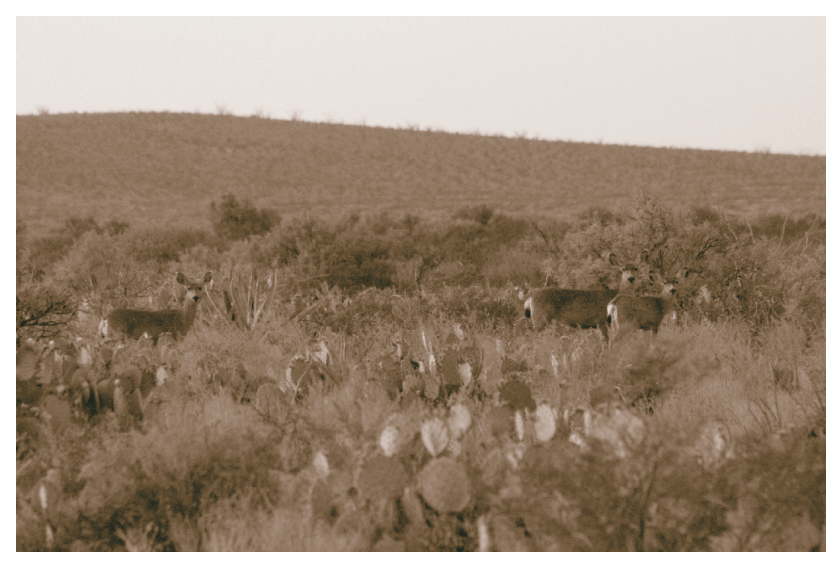

Figura 3. Venado bura (Odocoileus hemionus).

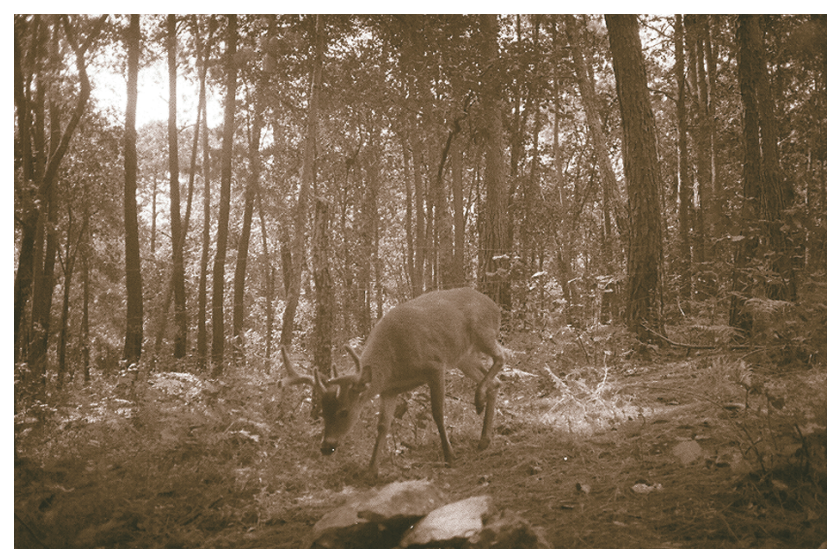

Figura 4. Venado colablanca (O. Virginianus).

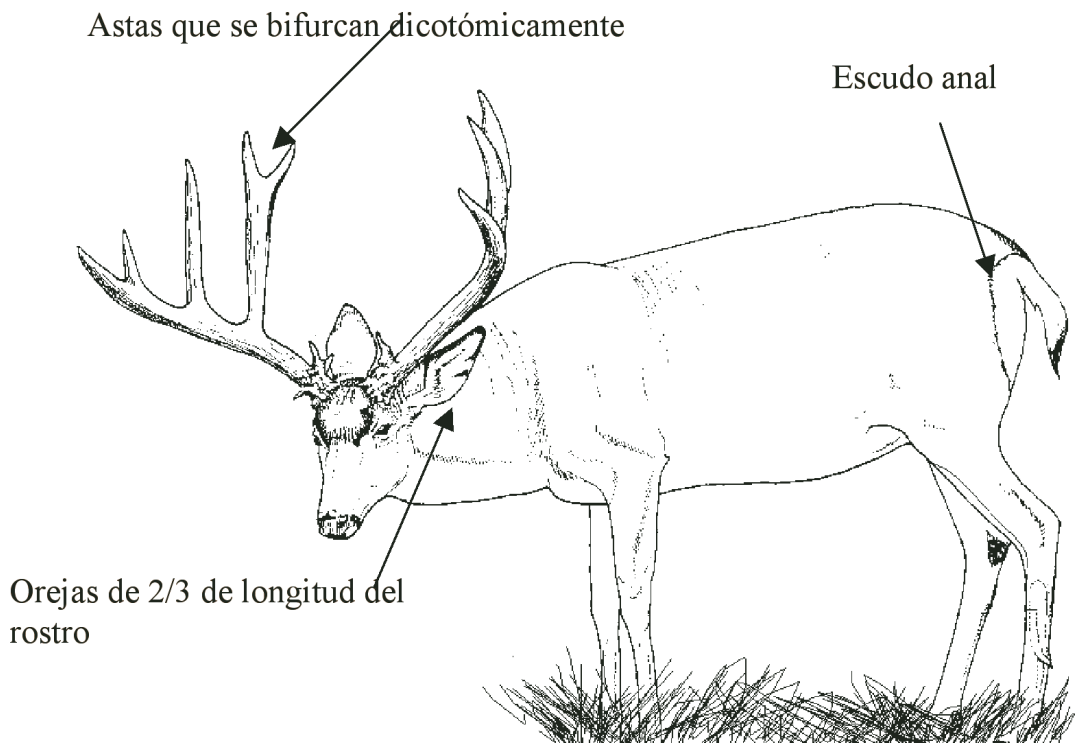

Figura 5. Macho de venado bura donde se marcan sus características más distintivas, como son el tener las astas que se bifurcan de forma dicotómica, las grandes orejas y la presencia de un escudo anal. 
rango y la región noreste de Sonora (ver Figura 6). Actualmente la situación en su rango de distribución es desconocida, pero existen registros que mencionan que en el estado de Nuevo León la especie se ha extinguido, al igual que las poblaciones del centro de Coahuila (DIETRICH, 1989; DIETRICH \& TIJERINA, 1988) aunque se han reintroducido individuos en algunas áreas de Nuevo León y Coahuila. Para el norte de Zacatecas la población se restringe a una pequeña zona aislada en el municipio de Villa de Cos (DÍAZ-CASTORENA, 1989). De esto resulta evidente que hay una reducción en su área de distribución tanto en su extremo sur como del lado oriental de la distribución que tenía en México.

La información sobre la abundancia de esta especie es escasa en México, pero en un trabajo donde se compara la información de las estimaciones poblacionales del venado bura publicadas durante el período de 1980-1990 en el Journal of Wildlife Management para la región del Desierto Chihuahuense dentro de los Estados Unidos, se encontró un promedio de 6.6 \pm 1.42 d.s. venados $/ \mathrm{km}^{2}$, lo cual es una densidad poblacional menor que la que tienen otras subespecies de bura (GALINDO-LEAL, 1993). Esto sugiere que estas poblaciones naturalmente son de densidades bajas y que se encuentran fragmentadas. En general, se considera que las poblaciones del venado bura han disminuido su rango de distribución geográfico en su distribución más sureña (que es la que corresponde a México), localmente se ignora el estado actual de las poblaciones del desierto Chihuahuense y, pese a eso, está sujeta a un aprovechamiento cinegético. Por lo

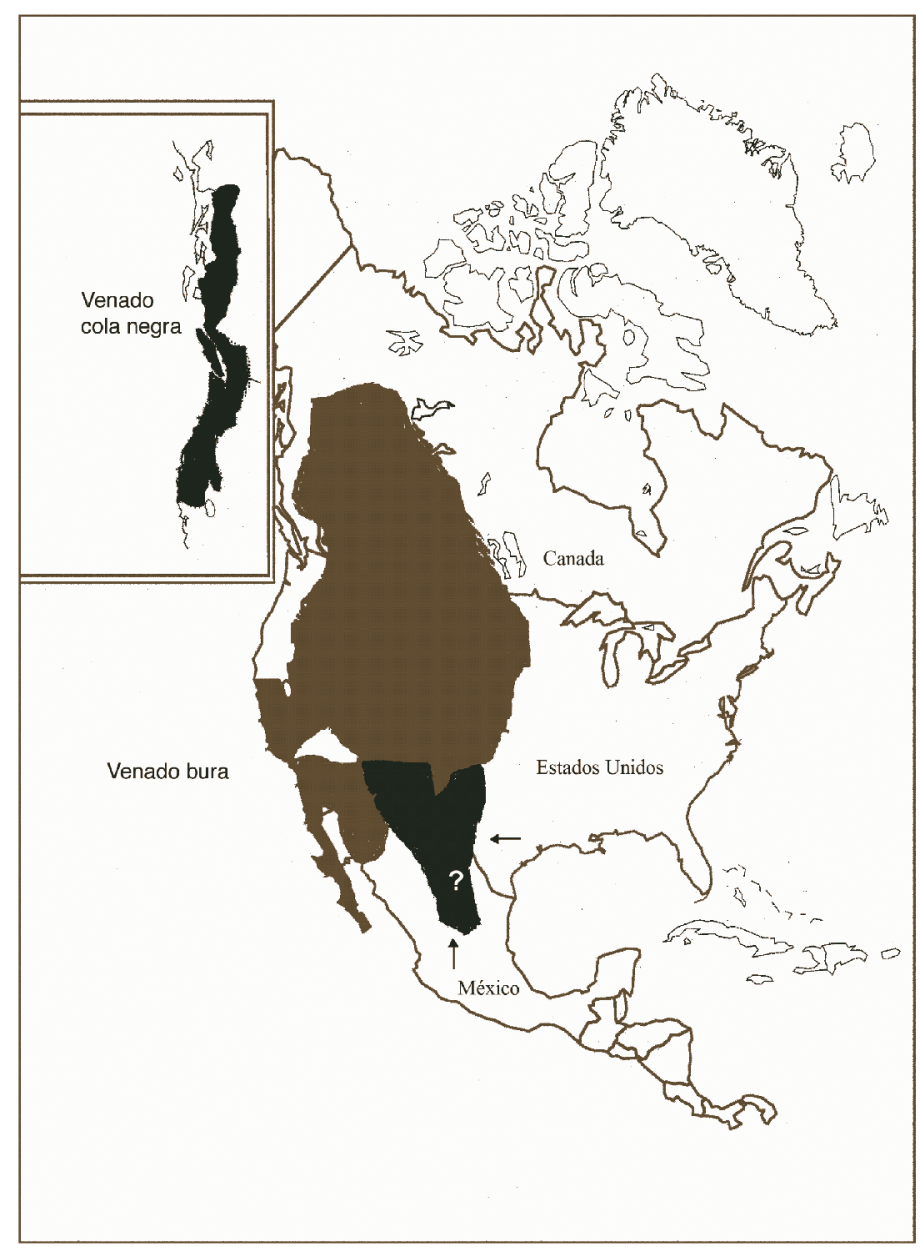

Figura 6. Distribución geográfica para la especie de venado bura o cola negra (Odocoileus hemionus), en Norteamérica. En negro se marca su rango de distribución en el desierto chibuahuense. La región sur de su distribución que perteneciente a México se marca con una signo de interrogación pues actualmente se desconocen los limites reales, aunque se sabe que ha disminuido de sur a norte y de este a oeste, como indican las flechas. 
tanto, resulta relevante estudiar aspectos biológicos de esta especie en el área protegida más sureña donde se encuentra, es decir en la Reserva de la Biosfera Mapimí y, al mismo tiempo, utilizar la información obtenida de esta especie para incorporarla a un plan de manejo de la reserva.

\section{EL VENADO EN LA RESERVA DE LA BIOSFERA MAPIMÍ}

En la Reserva de la Biosfera Mapimí se ha detectado que la población de los venados bura se encuentra espacialmente, estructurada teniendo fragmentos de hábitat adecuado para esta especie asociado a los elementos del paisaje (descritos por MONTAÑA \& BREIMER, 1988) que contienen sierras y/o montes aislados (SÁNCHEZ-ROJAS, 2000). Al comparar fragmentos del mismo elemento del paisaje se ha observado que la densidad de individuos no es uniforme, que es baja como en otras regiones del desierto chihuahuense, y que ésta puede variar en función de la calidad del fragmento. Se encontró que en los individuos de estas subpoblaciones el proceso de selección de hábitat hacia el interior de estos fragmentos indica que hay una mayor selección de lugares con mayor heterogeneidad del terreno, que suponemos está asociado a sus estrategias antidepredatorias, y además en la época de secas con la cercanía a las fuentes de agua; esta heterogeneidad puede estar asociada con otros factores tales como la densidad y diversidad vegetal los cuales pueden influir en la calidad del forraje (SÁNCHEZ-ROJAS \& GALLINA, 2000a; 2000b).

Tratando de establecer si había diferencias no sólo en la densidad sino en otros de los parámetros de la población, y basados en que la morfología del pellet (excreta) lo permite en otras especies de cervidos (BUBENIK, 1982; EZCURRA \& GALLINA, 1981; MACCRAKEN \& VAN BALLENBERGHER, 1987), se logró discriminar por el tamaño de los pellets a tres grupos (jóvenes, hembras adultas y machos adultos) que conforman las poblaciones del venado bura, utilizando muestras provenientes de animales en cautiverio (SÁNCHEZ-ROJAS et al., 2004). Tal como predice la dinámica de fuente-vertedero, los hábitats fuentes (con mejores condiciones de hábitat) tuvieron una mayor producción de juveniles, y en este caso también tuvieron una mayor densidad absoluta de individuos (SÁNCHEZ-ROJAS, 2000).

\section{LA CONSERVACIÓN DEL BURA EN MAPIMÍ}

Con esta información sobre la población de venado bura se puede visualizar cómo esta población puede integrarse a los criterios usados para establecer prioridades de conservación dentro de la reserva, pues generalmente se consideran tres criterios para establecer prioridades de conservación en un área natural protegida: a) singularidad: una comunidad biológica recibe mayor prioridad de conservación si está formada por especies raras o endémicas que si esta formada por especies comunes y de amplia distribución, a su vez, una especie tendrá un mayor valor de conservación si es taxonómicamente única que si tiene muchos taxones relacionados; b) estado de conservación: las especies en riesgo de extinción serán prioritarias sobre las especies que no lo están; y c) utilidad: las especies que tienen un valor (presente o potencial) para la poblaciones locales son de un valor de conservación mayor que las que no lo tienen (PRIMACK, 1993).

El venado bura no se ajusta al criterio de singularidad estrictamente ya que no es una especie endémica ni es una taxón único, aunque en muchos sistemas áridos sea el herbívoro nativo más grande que se presenta. Sin embargo, consideramos que el venado bura sí se puede ubicar en los otros dos criterios de prioridad, debido a que existe un riesgo de extinción de la especie y a que es una especie con un valor económico para las poblaciones de seres humanos que habitan la región de Mapimí.

Esta consideración de riesgo de extinción se basa en que las poblaciones de venado bura dentro de la parte mexicana del Desierto Chihuahuense, aún con la escasa información que existe muestra que se han presentado extinciones locales de las poblaciones lo que ha producido una disminución en el rango de distribución geográfica (Figura 6). Es muy posible que las poblaciones de venado bura dentro del Desierto Chihuahuense se comporten también como una metapoblación, por lo que haya un conjunto de subpoblaciones con bajas densidades, las cuales muy probablemente enfrentarán los problemas que presentan las poblaciones pequeñas, tales como la 
variación aleatoria de las tasas de natalidad y mortalidad; la variación temporal de las condiciones ambientales (tales como la depredación, las enfermedades, disponibilidad de alimento, catástrofes naturales) y la reducción de la variación genética. Estos problemas incrementan sensiblemente las posibilidades de extinción (CAUGHLEY \& GUNN, 1996). Por lo tanto, la permanencia de la especie dependerá de que haya subpoblaciones remanentes que aporten los individuos que mediante la dispersión pueden recolonizar los fragmentos de hábitat adecuado vacio.

Por lo que respecta al potencial económico del venado bura, al ser una especie sujeta a aprovechamiento cinegético, potencialmente es alto, y en algunos lugares como Sonora representa una de las especies con más individuos capturados. Dentro de las áreas naturales protegidas no es posible llevar a acabo esta actividad en las zonas núcleo, pero sí en las zonas de influencia y de amortiguamiento de la misma, siempre y cuando se cumplan con los requisitos que la legislación ambiental imponga.

La población de los venados bura debería de considerarse prioritaria en Mapimí y por tanto debería de ser monitoreada en largos periodos de tiempo. Dicho monitoreo deberá incorporar la estructuración espacial, poner énfasis en la dinámica que manifiesten las diferentes subpoblaciones, detectar si un fragmento mantiene su identidad de fuente o de vertedero a lo largo del tiempo, identificar qué factores se encuentran asociados a esta condición y si estos son constantes o no. Toda esta información nos permitirá, a largo plazo, tener planes de manejo de la especie para garantizar su permanencia.

\section{BIBLIOGRAFÍA}

ALVAREZ-BUYLLA, E. R., R. GRACÍA-BARRIOS, C. LARA-MORENO \& M. MARTÍNEZ-RAMOS. 1996. Demographic and Genetic Models in Conservation Biology: application and perspectives for tropical rain forest tree species. Annual Review of Ecology and Systematics 27:387-421.

ANDERSON, A. E. \& O. C. WALLMO. 1984. Odocoileus hemionus. Mammalian species 219: 1-9.

ANDREWARTHA, G. H. \& L. C. BIRCH. 1984. The ecological web. University of Chicago Press: Chicago Illinois.
BOORMAN, S. A. \& P. R. LEVITT. 1973. Group selection on the boundary of stable population. Theorical Population Biology 4: 85-128.

BUBENIK, R. A. 1982. Physiology. Pp 125-179. En: Thomas J. W. \& D. E. Toweill (eds). Elk of North America. Ecology and management. Stackpole Books, Harrisburg, PA.

CAUGHLEY, G. \& A. GUNN. 1996. Conservation Biology in Theory and Practice. Cambridge: Blackwell Sciences.

DÍAZ-CASTORENA, M. A. 1989. Distribución histórica y actual del venado bura (Odocoileus hemionus crooki) en el estado de Zacatecas. Memorias del III Simposio de Venados en México, pp. 108-110. Facultad de Medicina Veterinaria y Zootecnia UNAM: México, D. F.

DIETRICH, U. 1989. Reporte del avance sobre el proyecto de reintroducción del venado bura (Odocoileus hemionus crooki) en el estado de Nuevo León. Memorias del III Simposio de Venados en México, pp. 24-28. Facultad de Medicina Veterinaria y Zootecnia UNAM: México D.F.

DIETRICH, U. \& G. TIJERINA. 1988. Situación actual y perspectivas de la población del venado bura en el estado de Nuevo León y experiencia en el centro reproductivo de venado de la facultad de ciencias forestales (U.A.N.L.), Linares, Nuevo León. Memorias del II Simposio de Venados en México, pp. 18-32. Facultad de Medicina Veterinaria y Zootecnia UNAM: México D.F.

EZCURRA, E. \& G. GALLINA. 1981. Biology and population dynamics of white-tailed deer in northwestern Mexico. En: Folliott, P.F. \& Gallina, S. (Eds.). Deer Biology, Habitat Requirements, and Management in Western North America. México, D. F. Instituto de Ecología. Pp. 78-108.

FAHRIG, L. \& G. MERRIAM. 1994. Conservation of fragmented Populations. Conservation Biology 8: 50-59.

FARINA, A. 1998. Principles and methods in landscape ecology. Chapman \& Hall: London.

FORMAN, R. T. T. \& M. GODRON. 1986. Landscape ecology. Wiley and Sons: New York.

GALINDO-LEAL, C. 1993. Densidades poblacionales de los venados cola blanca, cola negra y bura en Norteamérica. En: R. A. Medellín \& G. Ceballos (eds.), Avances en el Estudio de los Mamíferos de México, Publicaciones Especiales 
Vol I, Asociación Mexicana de Mastozoología, México, D. F. Pp. 371-391.

GILPIN, M. E. \& I. HANSKI. 1991. Metapopulation dynamics: empirical and theorical investigation. Academic Press, London.

GÓMEZ-POMPA, A. \& R. DIRZO. 1995. Reservas de la biosfera y otras áreas naturales protegidas de México. Instituto Nacional de Ecología y Comisión Nacional para el Conocimiento y Uso de la Biodiversidad,México D. F.

HALFFTER, G. 1984. Las reservas de la biosfera: Conservación de la naturaleza para el hombre. Acta Zoológica Mexicana 5: 1-50.

HALFFTER, G. 1985. Biosphere reserve: Conservation of nature for man. PARKS 10: 15-19.

HALFFTER, G., C.E. MORENO, E.O. PINEDA. 2001. Manual para evaluación de la biodiversidad en reservas de la biosfera. M\&T Manuales y Tesis SEA, España. 80 pp.

HANSKI, I. \& M. GILPIN. 1991 Metapopulation dynamics: brief history and conceptual domain. Biological Journal of the Linnean Society 42: 3-16.

HANSKI, I. \& O. E. GAGGIOTTI. 2004. Genetics and Evolution of Metapopulations. Academic Press London. 629 pp.

HEFFELFINGERJ. 2000. Status of the name Odocoileus hemionus crooki (Mammalia: Cervidae). Proceedings of the Biological Society of Washigton. 113: 319-333.

LEOPOLD, S. 1965. FAUNA SILVESTRE DE MÉXICO. Instituto Mexicano de Recursos Naturales Renovables, México D. F.

LEVIN, S. 1992. The problem of pattern and scale in ecology. Ecology 73: 1943-1967.

LORD, J. M. \& D. A. NORTON. 1990. Scale and the spatial concept of fragmentation. Conservation Biology 4: 70-79.

MACCRAKEN, J. G. \& V. VAN BALLENBERGHER. 1987. Age-and-Sex Related differences in fecal pellet dimension of Moss. Journal of Wildlife Management 51: 360-364.

MCCULLOUGH, D. R. 1996a. Spatially structured populations and harvest theory. Journal of Wildlife Management 60: 1-9.

MCCULLOUGH, D. R. 1996b. Introduction. En: D. R. McCullough (ed.), Metapopulations and Wildlife Conservation. Island Press, Washington. Pp. 1-10.

MONTAÑA, C. \& R. F. BREIMER. 1988. Major vegetation and environment units. En: C. Monta- ña (Ed.), Estudio integrado de los recursos vegetación, suelo y agua en la reserva de la biosfera de Mapimí. México D. F. Instituto de Ecología. Pp. 99-114.

PRIMACK, R. B. 1993. Essentials of Conservation Biology. Massachusetts, Sinauer Associates.

PULLIAM, H. R. 1988. Source, sink and population regulation. American Naturalist 132: 652-661.

PULLIAM, H. R. \& B. J. DANIELSON. 1991. Sources sink and habitat selection: a landscape perspectives on population dynamics. The American Naturalist 137: S50-S66.

PULLIAM, H. R. \& J. B. DUNNING. 1994. Demographic processes population dynamics on heterogeneous landscape. En: G. K. Maffe \& C.R Carol (ed.). Principles of conservation biology. Sinauer Associates, Masachussetts. Pp. 179-205 SÁNCHEZ-ROJAS G. 2000. Conservación y manejo del venado bura en la reserva de la biosfera de Mapimi. Tesis de doctorado en Ecología y Manejo de Recursos Naturales Instituto de Ecología, A.C. México. pp. 78.

SÁNCHEZ-ROJAS G. \& G. GALLINA. 2000a. Factors affecting habitat use by mule deer (Odocoileus hemionus) in the central part of the Chihuahuan Desert, Mexico: an assessment with univariate and multivariate methods. Ethology Ecology and Evolution 12: 405-417.

SÁNCHEZ-ROJAS G. \& S. GALLINA. 2000b. Mule deer (Odocoileus hemionus) density in a landscape elements of the Chihuahuan desert, Mexico. Journal of Arid Environments 44: 357-368.

SÁNCHEZ-ROJAS, G., S. GALLINA \& M. EQUIHUA. 2004. Pellet morphometry as tool to distinguish age and sex in the mule deer. Zoo Biology 23: 139-146.

SPARROW, A. D. 1999. A heterogeneity of heterogeneities. TREE 14: 422-423.

SZACKI, J. 1999. Spatially structured population: how much do they match the classic metapopulation concept? Landscape Ecology 14: 369-379.

UNESCO. 1996. Reservas de la biosfera: La estrategia de Sevilla y el marco estatutario de la red mundial. UNESCO, Paris.

WALLMO, O. C. 1981. Mule and Black-tailed deer distribution and habitats. En: O. C. Wallmo (ed.) Mule and Black- tailed Deer of North America. University of Nebraska press, Nebraska. Pp. 366-386. 